\section{LA GESTIÓN DE GALIDAD COMO INNOVACIÓN ORGANIZACIONAL PARA LA PRODUCTIVIDAD EN LA EMPRESA}

FECHA DE RECEPCIÓN: 13 de mayo FECHA DE APROBACIÓN: 29 de julio Pp. 20-41

\section{QUALITY MANAGEMENT AS AN DRGANIZATIONAL INWOVATION FOR THE PRODUGTIVITY OF THE GOMPANY}

\author{
Luis Carlos Arraut Camargo*
}

* Doctor en Dirección de EmpresasUniversidad Mondragón (España); Ingeniero industrial-Universidad Tecnológica de Bolívar; Profesor de la Facultad de Ingenierías de la Universidad Tecnológica de Bolívar. Miembro del grupo de investigación de Gestión de la Innovación y el Conocimiento. 


\section{RESUMEN}

$E$

presente artículo desarrolla un tema de interés para académicos y empresarios como es el de la innovación. El tema central es la innovación de tipo organizacional a partir de los sistemas de calidad y su efecto en la productividad y competitividad de las empresas.

La metodología utilizada es de múltiples casos explicativos tomando como unidad de análisis el sector petroquímico-plástico de la ciudad de Cartagena de Indias.

Las principales conclusiones que aquí se presentan, demuestran primero la importancia de un fenómeno organizacional como es la innovación organizacional y cómo un grupo de empresas manufactureras de la ciudad de Cartagena de Indias, desarrollan este tipo de innovación a partir de sus sistemas de calidad ISO con un efecto positivo en la productividad de las mismas.

Este artículo es producto del trabajo doctoral del autor sobre la innovación de tipo organizacional en el sector petroquímico-plástico de la ciudad de Cartagena de Indias.

\section{ABSTRACT}

$T$ his article shows the importance of innovation for academic professionals and company managers. The main topic is organizational innovation based on quality systems and their impact on productivity and competitiveness.

The methodology used is that of explained multiple cases taken as a unit of analysis in the petrochemical-plastic sector in Cartagena city, Colombia.

The principal results are, first, the importance of organizational innovation and how a group of manufacturing companies in Cartagena, Colombia, develops this kind of innovation based on quality systems ISO as a positive effect on their productivity.

This paper is the result of a doctoral degree research on organizational innovation in the petrochemical-plastic sector in Cartagena, Colombia.

\section{RESUMÉÉ}

Cet article développe un sujet d'intérêt pour les académiciens et les chefs d'entrepris il traite de l'innovation. Le sujet central en est l'innovation organisationnelle des systèmes de qualité et son effet sur la productivité et la compétitivité des entreprises. La méthodologie utilisée s'appuie de multiples cas explicatifs et prend comme unité d'analyse le secteur pétrochimique-plastique de la ville de Carthagène des Indes. Les conclusions principales qui en sont tirées, démontrent d'abord l'importance du phénomène de l'innovation organisationnelle. Elles montrent de plus comment un groupe d'entreprises manufacturières de la ville de Carthagène des Indes, développant ce type d'innovation à partir du système de qualité ISO, a un effet positif sur la productivité de ces dernières. Cet article est le produit d'un travail de doctorat sur l'innovation organisationnelle dans le secteur pétrochimique-plastique de la ville de Carthagène des Indes.

\section{Palabras claves}

Innovación

Innovación organizacional

Productividad

Calidad

Competitividad

Sistemas de calidad

\section{$\Rightarrow \quad$ Key words}

Innovation

Organizational Innovation, Productivity

Quality

Competitiveness

Quality systems $\rightarrow \quad$ Mots clefs

\author{
Innovation \\ Innovation \\ organisationnelle \\ Productivité \\ Qualité \\ Compétitivité \\ Systèmes de qualité
}




\section{INTRODUCGIÓN}

eniendo en cuenta que hoy en día la innovación es

el factor dominante en el crecimiento económico de una nación o región y en los patrones de comercio internacional, convirtiéndose en la fuerza motriz que impulsa a las empresas hacia objetivos ambiciosos a largo plazo y que conduce a la renovación de las estructuras organizacionales, este artículo presenta el resultado de un trabajo de investigación en el tema de la innovación de tipo organizacional.

Cuando se hace referencia a la innovación de tipo organizacional es necesario tener en cuenta, para su estudio en las empresas, la interrelación de diferentes componentes. Estos, son elementos necesarios para aumentar la capacidad innovadora de las organizaciones.

El objetivo de este trabajo es presentar un análisis de la innovación organizacional y su relación con los sistemas de calidad en las empresas, tomando como unidad de análisis empresas del sector petroquímico-plástico de la ciudad de Cartagena de Indias.

Para conseguir este objetivo se presentan cuatro apartados, así: el primero hace una revisión del concepto de innovación organizacional, su importancia en el contexto de la empresa. El segundo, presenta la metodología de casos múltiples explicativos (CME), las preguntas de investigación y las preposiciones de estudio. En el tercero se abordan los resultados de la forma en que los sistemas de calidad como innovación organizacional, mejoran la productividad de las empresas de estudio. Por último, se plantean las conclusiones del estudio.

Este trabajo aporta a los académicos nuevas luces en el estudio de la innovación y un aporte a la clase empresarial para entender de mejor forma en la práctica, el fenómeno de la innovación. 


\section{LA INNOVACIÓN}

\subsection{CONCEPTOS}

La innovación toca dos aspectos importantes, uno es el comercial y el otro la creatividad, lo anterior puede dar pie a un concepto interesante sobre innovación de acuerdo con Morcillo (1997): "Viendo lo que todo el mundo ve, leyendo lo que todo el mundo lee, oyendo lo que todo el mundo oye, innovar es realizar lo que nadie ha imaginado todavía”.

Mientras el invento es la creación de una nueva idea, la innovación es un concepto más amplio, pues comprende el proceso de desarrollo y aplicación de una nueva idea. Esta puede representar una recombinación de ideas viejas, un esquema que cuestiona el orden actual, una fórmula o un enfoque singular que sus proponentes perciben como nuevo (Van de Ven, 2001).

Es claro que no existe innovación sin la adopción de la idea aunque no sea nueva para la organización que la adopta o tenga un éxito o aceptación comercial. Generar buenas ideas o adoptar una nueva, en sí misma, o por sí misma, es solo el comienzo. Para ser una innovación, se tiene que convertir una idea en un producto o servicio que deseen los clientes. Presentarse con la idea o el prototipo es una invención. Protegerla y fomentarla hasta que se convierta en un producto y/o servicio con un mercado de clientes potenciales es otra cosa.

E1 CIDEM $(2004,10)$ en su guía para gestionar la innovación, define la innovación como:

"Todas aquellas tareas relacionadas con hacer cosas nuevas (diseño y desarrollo de nuevos productos) y con hacer las cosas de manera diferente para aumentar el valor de los productos (redefinición de los procesos empresariales). El proceso de innovación ha de estar marcado por un enfoque de mercado muy claro. En efecto, se inicia con un input del mercado: la detención de una oportunidad o necesidad insatisfecha; y finaliza también en el mercado con un output la satisfacción de los clientes por el nuevo producto o servicio creado".
La innovación es una disciplina que han de practicar todas las empresas, independientemente de su dimensión y del sector en que compiten.

Algunos países como Gran Bretaña definen la innovación como la explotación sucesiva de nuevas ideas y como eje importante de desafío en la economía del

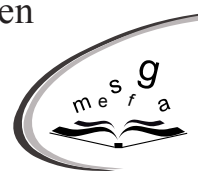
conocimiento. La innovación tiene que ver con productos y procesos, además de nuevas vías de hacer negocio (Gran Bretaña, Reporte de la Innovación 2003).

En el año 2003, en el Libro Verde del Sistema Nacional de Innovación Español se plantea que el conocimiento puede ser tecnológico o igualmente de mercado o de una mejor organización. Se habla por lo tanto en este documento que existen tres tipos de innovaciones: de mercado, organizativas y por supuesto tecnológica (producto o proceso). Igualmente, en el año 1995 la Comisión Europea en su Libro Verde de la Innovación, diferenciaba en forma clara cuatro tipos de innovación: en los procedimientos, en productos (o servicios), en la organización del trabajo y en acceso a mercados.

Es claro cómo el concepto de innovación ha evolucionado; instituciones como la OCDE (Organización para la Cooperación y Desarrollo) amplía el concepto enfocándolo no sólo como innovación tecnológica, es decir, de nuevos productos y procesos implementados o introducidos en nuevos mercados (Manual de Oslo, 1992, 1997 y Manual de Frascati,1993), sino considerando los aspectos de mercados y organizacionales.

En el Manual de Oslo (1992,1997), se define el con-cepto de innovación enfocada a la implementación tecnológicamente de nuevos productos y procesos, además del mejoramiento tecnológico de productos y procesos. Estas innovaciones tienen que estar implementadas e introducidas en el mercado y se denominan innovaciones tecnológicas de producto y proceso (TPP). Incluye actividades científicas, financieras, organizacionales y de mercado. 


\section{La innovación es una disciplina que han de practicar todas las empresas, independientemente de su dimensión y del sector en que compiten.}

El Manual de Oslo (2005), desde el punto de vista del concepto de innovación tiene en cuenta que una empresa puede realizar múltiples innovaciones en sus métodos de trabajo, en el uso de los factores y en los procedimientos de producción para mejorar su productividad y/o funcionamiento comercial. Frías, (2006) define la innovación como:la implementación de un nuevo o mejorado producto y/o servicio (good or service), o proceso, un nuevo sistema de marketing, o un nuevo método organizacional como prácticas de negocio, organización del trabajo y relaciones externas. Los requerimientos mínimos para una innovación es que el producto, proceso, sistemas de marketing o método organizacional sea nuevo o significativamente nuevo para la empresa. Esto incluye productos, procesos y métodos desarrollados por estas; también pueden ser adoptados por otras firmas y organizaciones.

Resumiendo lo anterior, es claro que hoy en día el concepto de innovación implica:

$\Rightarrow$ La aceptación en el mercado.

$\Rightarrow$ Factores tecnológicos y no tecnológicos.

$\Rightarrow$ Proceso de gestión de la innovación en la organización,

$\Rightarrow$ Utilización de herramientas y técnicas de gestión de la innovación,

$\Rightarrow$ Modelo de gestión de la innovación que involucre a todas las áreas de la organización.

\subsection{LA INNOUACIÓN Y LA GESTIÓN DE LA BADIID}

El papel que juega la innovación como factor de primer orden en el desarrollo socio-económico de las regiones es una prioridad en el mundo actual.

Algunos académicos han llegado a establecer la relación de la calidad y la innovación como dos conceptos centrales de nuevas teorías económicas de la empresa, o como modelos de comportamiento empresarial en el ámbito normativo (Perdomo, 2010).

El papel de la innovación y el desarrollo tecnológico sobre la productividad y la competitividad de las empresas, el bienestar de la sociedad y el progreso de los países, han despertado el interés de los investigadores de diferentes ámbitos que han realizado estudios sobre los factores que afectan la innovación.

El proceso de internacionalización de las economías, producto de los mercados globalizados, realza la importancia de la productividad industrial. Para competir en mercados de una economía global se requiere un amplio conocimiento del entorno, de una óptima capacidad de gestión de la innovación, de una adecuada infraestructura tecnológica y de un talento humano capacitado.

En la actualidad, las empresas no pueden considerar la innovación como un evento ocasional. Si una empresa no es capaz de transformar sus productos, su forma de producción, manejar modelos de gestión y estructuras flexibles en un contexto de incertidumbre, no será capaz de sobrevivir.

En la literatura universal la relación entre la calidad y la innovación no ha sido un tema recurrente de investigación ni ha sido un tema tratado directamente por investigadores y académicos.

Las argumentaciones teóricas encontradas se centran en dos aspectos del análisis: primero, considerar los sistemas de calidad como plataformas propicias para el desarrollo de las innovaciones; y segundo, cuando se muestran indirectamente algunos resultados de la innovación; están o pueden ser influenciados por el sistema de calidad de la empresa. 
La innovación, como una actividad estratégica, se observa en la importancia que juega en la estrategia de la empresa; su capacidad depende de la forma en que esta se estimule dentro de la organización. Esto permite la generación de ideas (cartera de ideas), de proyectos (banco de proyectos) y el desarrollo de inventos o mejoras y su posterior lanzamiento exitoso (éxito comercial). Innovar no sólo es la generación de ideas, es necesario transformarlas en productos y/o servicios para que sean valoradas positivamente por el mercado, lo que garantiza el éxito comercial.

\section{3 ¿QUÉ ES LA INNOUACIÓN DRGANZACONAL? ¿EUAL ES SU IMPORTANOIA EN EL BONTEXTO}

La innovación en términos generales tiene gran importancia para el crecimiento micro y macro, como se ha venido promulgando a través de estudios académicos y la opinión de directivos de las empresas de diferentes países. Igualmente, se manifiesta su importancia por los diferentes programas y proyectos entre regiones que se han venido desarrollando en este tema.

Esta importancia también la podemos señalar de acuerdo con el beneficio que implica la innovación para diferentes sectores de la sociedad, por ejemplo (Sistema Nacional de Innovación de la Gran Bretaña; 2003):

$\Rightarrow$ Para los clientes y/o consumidores de productos y/o servicios, es importante porque desarrolla productos de alta calidad y valor agregado para el consumidor; mejora la eficiencia del servicio (público o privado) y la calidad de vida.

$\Rightarrow$ Para las empresas, la innovación ayuda a sostenerse o mejorar su crecimiento; conlleva beneficios económi-

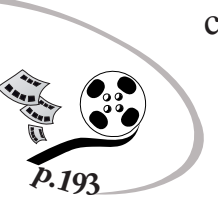
cos para sus propietarios e inversionistas.

Para los empleados, la innovación hace más interesante o atractivo su trabajo, mejorando sus habilidades y el salario.
Igualmente en ausencia de la innovación puede llevar a estancamiento de las empresas y pérdida de trabajo.

$\rightarrow$ Para la economía, es la clave para la alta productividad y la gran prosperidad de todos. Es importante en todos los sectores de la economía y en las empresas tanto manufactureras como de servicio.

También se considera, a juicio de investigadores norteamericanos, que la innovación organizacional es el principal motivo de la ventaja tecnológica y competitividad de Estados Unidos, desde los años sesenta.

La innovación generalmente ha sido vinculada con la tecnología; así, se ha analizado y estudiado el tema como innovación tecnológica. Pero en los últimos años ha venido tomando fuerza el concepto de innovación no tecnológica (Organizacional y Marketing).

Un aspecto que resalta la importancia en los últimos años de la innovación no tecnológica, lo encontramos en el estudio Marcador de Innovación Europeo (European Innovation Scoreboard - IES) ${ }^{1}$, en el que a partir del 2004 esta se evalúa y se analizan los siguientes factores:

$\Rightarrow$ El cambio no técnico.

$\Rightarrow$ La mejora de las estructuras.

$\Rightarrow$ La puesta en práctica de técnicas de dirección avanzada.

$\Rightarrow$ Los cambios importantes en cuanto a diseño de productos o servicios.

Este artículo resalta la importancia de la innovación no tecnológica y deja entrever que no desarrollar este tipo de innovaciones puede impedir el mejoramiento de la capacidad de innovación de las empresas de la Unión Europea con respecto a las empresas de Estados Unidos y Japón.

Los estudios indican que la ventaja de EE.UU. sobre la UE en términos de crecimiento de la productividad no se basa solamente en la innovación tecnológica. La innovación no técnica puede ser el eslabón perdido que impide a Europa aprovechar completamente las nuevas oportunidades tecnológicas.

\footnotetext{
${ }^{1}$ Este estudio se realiza anualmente a partir del año 2000. Mide la capacidad innovadora de las empresas de los estados miembros de la Unión Europea con respecto a Estados Unidos y Japón. Para ampliar la información ingrese a http://www.trendchart.org/scoreboards/scoreboard2004/ index.cfm.
} 
Las innovaciones organizacionales (reducción de planos jerárquicos, delegación de ciertos niveles de toma de decisiones a nivel operativo) constituyen el requisito indispensables para el nuevo concepto de organización (Hammer, 1990).

En la actualidad, los avances en el sector de la informática y las telecomunicaciones ayudan a establecer la infraestructura necesaria para habilitar la innovación y la reinvención de los modelos de negocio (López et al., 2004), mediante la aplicación de la innovación organizacional.

Dentro de los principales cambios que muestra el Manual de Oslo (OCDE, 2005), se encuentran los siguientes (Sánchez y Castrillo; 2006):

$\Rightarrow$ Revisión y ampliación de la definición de innovación.

$\Rightarrow$ Revisión en la definición de actividades de innovación.

$\Rightarrow$ Nuevas consideraciones sobre las relaciones en el sistema de innovación.

La edición 2005 del Manual de Oslo amplía el concepto de innovación e incluye la innovación no tecnológica (organizacional y marketing). Lo anterior, debido a los crecientes estudios que señalan la importancia de los factores no tecnológicos en los procesos de innovación.
La innovación organizacional se considera importante en el crecimiento de las actividades innovadoras de la empresa. La expansión de las del sector servicio, los modelos de negocio construidos sobre nuevos métodos organizacionales, son innovaciones significativas. Incluso, cuando el uso de nuevas tecnologías está involucrada, el problema radica en identificar si es o no de naturaleza tecnológica (Lengrand, 2002).

Finalmente, la importancia de la innovación organizacional se puede apreciar en el Manual para la Normalización de Indicadores de Innovación Tecnológica en América Latina, al que se ha denominado Manual de Bogotá (2001 y 2004). Este manual establece que para una adecuada gestión de la innovación en las empresas, es importante tener en cuenta los aspectos organizacionales.

La adopción de la innovación no es solo técnica. Para ser exitosa, requiere activos complementarios que no siempre están presentes en las firmas. La incorporación de nuevas tecnologías exige cambios organizacionales importantes en las empresas. Anota este manual, que es importante indagar sobre la adopción de los métodos de trabajo y producción asociados con el toyotismo ${ }^{2}$, los cuales van más allá de los conocidos sistemas justo a tiempo y control o gestión de la calidad.

En la revisión a este manual en 2004, los expertos latinoamericanos que establecen los parámetros de este documento, incluyen dentro de la encuesta de medición, la innovación organizacional.

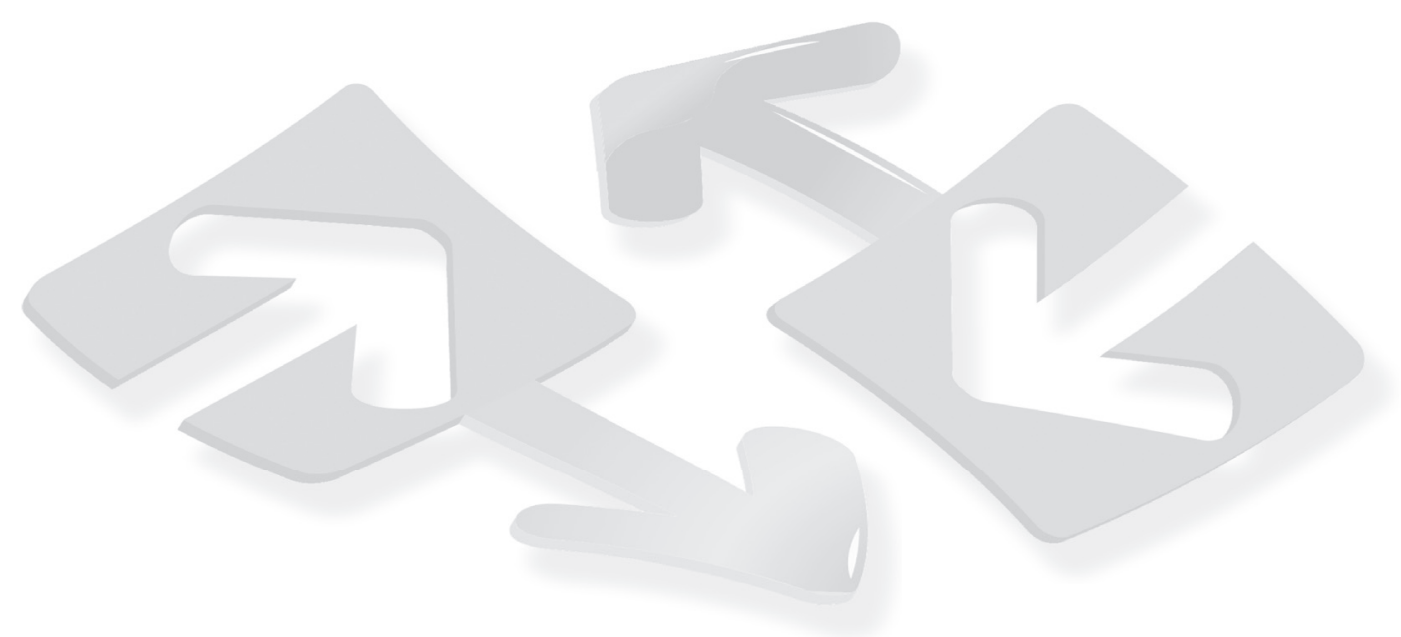

\footnotetext{
${ }^{2}$ Representa un modelo de producción industrial japonés basado en el trabajo flexible, aumento de la productividad a través de la gestión y organización (justo a tiempo).
} 


\section{MEtODOLOGía}

El trabajo de investigación realizado para este artículo utilizó el estudio de casos como herramienta metodológica teniendo en cuenta lo siguiente:

$\Rightarrow$ La metodología de casos es muy utilizada en la literatura de estudio de la organización (Bonache Pérez, 1999, p.123).

$\rightarrow$ Es la estrategia más adecuada cuando se busca responder el comó y el porqué; cuando el investigador tiene poco control sobre los hechos y acontecimientos y cuando nos centramos en un fenómeno contemporáneo (Yin, 1984, p13).

$\Rightarrow$ La complejidad del fenómeno a estudiar.

$\rightarrow$ Su aplicación como metodología de investigación se ha intensificado (Yin, 1994).

Con el fin de construir o depurar la teoría de la innovación de tipo organizacional (fenómeno organizativo) ${ }^{3}$, se utilizó la metodología de múltiples casos explicativos.

\subsection{METODOLOGÍA DE LA INVESTIGA- CIÓN: ESTUDIO DE MÚLTIPLES CASOS EXPLICATIVOS}

Siguiendo lo que se persigue en esta investigación se presenta la fase de metodología escogida.

\subsubsection{Modelo teórico preliminar}

El punto de partida estará determinado por la literatura sobre la innovación de tipo organizacional y la relación con los sistemas de calidad en búsqueda de la productividad y competitividad. En esta fase se trabajaron dos aspectos importantes. El primero fueron las preguntas de investigación:
$\Rightarrow$ ¿Cuáles son los factores internos y externos que condicionan los niveles de innovación organizacional en las empresas petroquímicas-plásticas de Cartagena de Indias?

$\Rightarrow \quad ¿$ Cuáles son las principales técnicas de gestión moderna que implican innovación organizacional y que desarrollan las empresas de estudio de Cartagena de Indias?

El segundo aspecto importante en esta fase de la investigación son las preposiciones (hipótesis) de estudio. Estas se presentan a continuación:

P1: Existe relación positiva entre la innovación organizacional y los sistemas de calidad en las empresas del sector petroquímico-plástico de Cartagena de Indias.

\subsubsection{Unidad de análisis}

La unidad de análisis escogida son las empresas del sector petroquímico-plástico de la ciudad de Cartagena de Indias, teniendo en cuenta su importancia en los sectores productivos de la ciudad y su grado de organización a nivel de grupos de empresas.

\subsubsection{Técnicas de recolección de información}

La recolección de información se realizó mediante encuesta y entrevista con los directivos de las empresas seleccionadas e igualmente se tuvieron en cuenta otras fuentes como informes de las empresas, literatura sobre los temas de estudio, información en web corporativa, entre otras.

\subsubsection{Análisis conjunto de la información}

Una vez se recopiló la información, se siguieron los pasos indicados para su análisis:

\footnotetext{
${ }^{3}$ El fenómeno organizativo en este trabajo hace referencia a la innovación y su relación con los sistemas de calidad en las empresas, especialmente las utilizadas en la unidad de análisis.
} 
$\Rightarrow$ La redacción y revisión de los informes preliminares por parte del autor, personas involucradas en el estudio, personas que trabajan o conocen el sector y académicos, entre otros.
$\Rightarrow$ El análisis en conjunto de la información, la extracción de conclusiones y validación.

En la tabla 1 se exponen los aspectos metodológicos más importantes, en la ficha técnica de la investigación.

\section{Tabla 1. Ficha técnica de la investigación}

\begin{tabular}{|c|c|}
\hline Metodología de investigación & Estudio de casos múltiple explicativos \\
\hline Unidad de análisis & $\begin{array}{l}\text { Empresas del sector petroquímico-plástico de Cartagena de Indias } \\
\text { (Colombia), ubicadas dentro de las } 200 \text { empresas más grandes de la } \\
\text { ciudad, teniendo en cuenta factores como ventas, utilidad, patrimonio. } \\
\text { Líderes en el mercado nacional y latinoamericano. }\end{array}$ \\
\hline Ámbito geográfico & Cartagena de Indias (Colombia-Sur América) \\
\hline Universo & Sector Petroquímico-Plástico de Cartagena de Indias \\
\hline Tipo de muestra & $\begin{array}{l}\text { Muestra lógica y teórica (capacidad de generalización analítica } \\
\text { del fenómeno estudiado), no de forma aleatoria (muestreo y } \\
\text { generalización estadística) }\end{array}$ \\
\hline Muestra & Cuatro empresas \\
\hline Método de recogida de la evidencia & $\begin{array}{l}\text { Literatura existente sobre el tema de investigación y conocimiento } \\
\text { de casos exitosos en el tema de investigación. } \\
\text {. Investigación exploratoria. } \\
\text { Revisión de documentos. } \\
\text { Realización de encuesta estructurada. } \\
\text { Realización de entrevistas semiestructuradas presencial. } \\
\text { Observación directa en visita a las empresas. } \\
\text {. Participación en reuniones de trabajo con empresas del sector. }\end{array}$ \\
\hline Fuentes de información & $\begin{array}{l}\text { Internas: documentación (memorias, informes y estudios internos), } \\
\text { páginas web, archivos de presentaciones. } \\
\text { Externas: publicaciones especializadas, congresos. }\end{array}$ \\
\hline Informadores clave & $\begin{array}{l}\text { Directos: directivos (mínimo cuatro) de las empresas del sector } \\
\text { estudiado de áreas claves en el tema de innovación. } \\
\text { Indirectos: personas involucradas en el sector como gerentes de } \\
\text { otras empresas que no están en el estudio, académicos especializados } \\
\text { en el tema de innovación, directivos de otras empresas del sector; } \\
\text { ingenieros de áreas claves de las empresas estudiadas. }\end{array}$ \\
\hline Métodos de análisis de la evidencia & $\begin{array}{l}\text { De tipo cualitativo: } \\
\text { - Identificación y enumeración estructural de la innovación } \\
\text { organizacional. (Modelos teórico preliminar). } \\
\text { - Identificar situaciones que permitan identificar innovaciones de } \\
\text { tipo organizacional y cómo se dan. } \\
\text { - Informes preliminares de cada caso con los informadores directos. } \\
\text { - Modelo inducido. } \\
\text { - Análisis de resultados y conclusiones. } \\
\text { De tipo cuantitativo: } \\
\text { - Análisis de clúster. }\end{array}$ \\
\hline Enfoque científico & $\begin{array}{l}\text { Inducción analítica a través de la lógica de la réplica (generalización } \\
\text { analítica). Proceso deductivos en la medida que se parte de } \\
\text { proposiciones de estudio de la revisión de la teoría. }\end{array}$ \\
\hline $\begin{array}{l}\text { Evaluación del rigor y calidad } \\
\text { metodológica }\end{array}$ & Validez y fiabilidad. \\
\hline Fecha de finalización & Mayo 2004-Febrero 2010 \\
\hline
\end{tabular}

Fuente. Elaboración propia. 


\section{LA INNOUAGIÓN ORGANIZAGIONAL Y SU RELAGIÓN CON LA PRODUGTIVIDAD Y COMPETIVIDAD}

a productividad y competitividad en las empresas está afectada por diferentes factores; para la presente investigación se ha buscado su relación con la innovación basado en los elementos claves de la capacidad innovadora de las empresas y sus resultados en su productividad.

Las empresas que conforman la unidad de análisis desarrollan prácticas innovadoras de tipo organizacional que les ha permitido mejorar su productividad y competitividad. En este apartado se presenta una forma de identificar la relación de innovación organizacional con la productividad y competitividad así: análisis del impacto de los sistemas gerenciales de calidad basados en la norma ISO 9001:2000 en las empresas de la unidad de análisis de acuerdo con informes de calidad ${ }^{4}$.

\subsection{LA INNOVACIÓN ORGANIZACIONAL [SISTEMA DE GESTIÓN DE CALIDAD] Y SU RELACIÓN CON LA PRODUCTI- TIVIDAD Y COMPEIITIVIDAD}

El tipo de innovación organizacional desde el punto de vista de la mejora en la práctica de la organización más tradicional y muy común en todos los casos estudiados

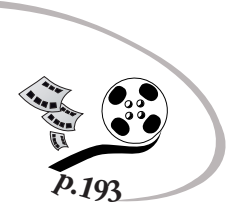

tiene que ver con los sistemas de calidad $^{5}$ de las empresas basado en la norma ISO 9000:2000, y es importante porque además la aplicación y mantenimiento de esta norma se convierte en un campo fértil para la innovación en la empresa. Para el presente análisis se tomaron variables e indicadores que las empresas utilizan para medir el impacto del Sistema de calidad $^{6}$. Las empresas en estudio constatan su sistema de indicadores, lo han perfeccionado con el tiempo y siempre están en constante cambio, por el mejoramiento continuo de sus procesos.

Los sistemas de medición de cada una de las empresas de estudio están integrados por indicadores financieros y no financieros. Además, establecen relaciones causales entre ellos y son utilizados por su equipo directivo; permiten proporcionar información eficiente y eficaz, que les posibilita por una parte, estar informados de la situación de la empresa $y$, por otra, actuar de manera inmediata para ajustar metas y objetivos estratégicos, en caso de no haber coincidencia con lo esperado.

Dentro de los indicadores que miden el impacto de la nor$\mathrm{ma}^{7}$ ISO 9000 en las empresas se tienen en cuenta variables de entrada, salida y proceso; además, están vinculados con los objetivos de la empresa y cumplen con las funciones de coordinación, seguimiento, control e intervención.

En conclusión, la capacidad de una empresa para competir en los mercados depende de sus relaciones con dos fuentes de ventaja competitiva: la calidad y la innovación. En la tabla 2, se muestra el resultado del indicador: servicio al cliente, conformado por tres criterios que son: nuevos clientes, \% de reclamos y \% de participación en el mercado. En cada uno de los tres criterios del indicador el sistema ISO 9000 de las empresas se tiene un impacto positivo en las empresas de estudio.

Se destaca en nuevos clientes (40\%) y disminución (24\%) de porcentajes de reclamos por parte de sus clientes en la empresa BFM. Por otra parte, en el criterio de mayor participación en el mercado se destaca la empresa PPC (30\%).

\footnotetext{
${ }^{4}$ La unidad de análisis o de estudio fueron cuatro empresas del sector petroquímico-plástico de la ciudad de Cartagena de Indias. Se han identificado con la iniciales, siguientes: PPC, BFM, PLB y CLX.

${ }^{5}$ Sistema de calidad en está investigación se refiere a la estructura organizacional, los procedimientos, los procesos y los recursos necesarios para la implementación de la calidad en las organizaciones.

${ }^{6}$ Esta información fue extraída de los informes de calidad suministrados por los responsables de la norma ISO 9000 en cada una de las empresas de la unidad de análisis.

${ }^{7}$ Para la escogencia de los indicadores que evalúan la estrategia de los sistemas de calidad en las empresas en estudio, se escogieron indicadores comunes a cada una y que estuvieran relacionados directamente con la actuación de las empresas (productividad y competitividad) de acuerdo con los objetivos de esta investigación, y con el fin de guardar uniformidad para el análisis de la investigación.
} 
Tabla 2. Efecto de la innovación organizacional en el servicio al cliente

Fuente. Elaboración propia

\begin{tabular}{|c|c|c|c|c|}
\hline Indicador & \multicolumn{4}{|c|}{ SERVICIO AL CLIENTE } \\
\hline \multicolumn{5}{|c|}{ EFECTO (\%) } \\
\hline \multirow[t]{5}{*}{ Nuevos clientes } & Empresa & Incremento & Disminución & Permanece \\
\hline & PPC & 30 & & \\
\hline & BFM & 40 & & \\
\hline & PLB & 30 & & \\
\hline & CLX & 55 & & \\
\hline \multicolumn{5}{|c|}{ EFECTO $(\%)$} \\
\hline \multirow[t]{5}{*}{$\%$ de reclamos } & Empresa & Incremento & Disminución & Permanece \\
\hline & PPC & & 15 & \\
\hline & BFM & & 24 & \\
\hline & PLB & & 15 & \\
\hline & CLX & & 20 & \\
\hline \multicolumn{5}{|c|}{ EFECTO $(\%)$} \\
\hline \multirow[t]{5}{*}{$\begin{array}{l}\% \text { de Participación } \\
\text { en el mercado }\end{array}$} & Empresa & Incremento & Disminución & Permanece \\
\hline & PPC & 30 & & \\
\hline & BFM & 22 & & \\
\hline & PLB & 10 & & \\
\hline & CLX & 25 & & \\
\hline
\end{tabular}

La tabla 3, muestra los resultados del indicador calidad en los procesos, conformado por tres criterios que son: eficiencia en procesos, eficacia en proceso y productividad de mano de obra. En cada uno de los tres criterios del indicador el sistema ISO 9000 hay un impacto positivo en las empresas de estudio. Se destaca en los tres criterios que conforman el indicador, la empresa BFM con los siguientes resultados: eficiencia en procesos $(52 \%)$, eficacia en procesos $(46 \%)$ y productividad de la mano de obra $(58 \%)$.

Tabla 3. Efecto de la innovación organizacional en los procesos

\begin{tabular}{|c|c|c|c|c|}
\hline Indicador & \multicolumn{4}{|c|}{ CALIDAD EN LOS PROCESOS } \\
\hline \multicolumn{5}{|c|}{ IMPACTO $(\%)$} \\
\hline \multirow[t]{5}{*}{ Eficiencia procesos } & Empresa & Incremento & Disminución & Permanece \\
\hline & PPC & 40 & & \\
\hline & BFM & 52 & & \\
\hline & PLB & 15 & & \\
\hline & CLX & 40 & & \\
\hline \multicolumn{5}{|c|}{ IMPACTO } \\
\hline \multirow[t]{5}{*}{ Eficacia en procesos } & Empresa & Incremento & Disminución & Permanece \\
\hline & PPC & 28 & & \\
\hline & BFM & 46 & & \\
\hline & PLB & 20 & & \\
\hline & CLX & 36 & & \\
\hline \multicolumn{5}{|c|}{ IMPACTO } \\
\hline \multirow[t]{5}{*}{ Brodłtgtividad mano } & Empresa & Incremento & Disminución & Permanece \\
\hline & PPC & 35 & & \\
\hline & BFM & 58 & & \\
\hline & PLB & 20 & & \\
\hline & CLX & 45 & & \\
\hline
\end{tabular}

Fuente. Elaboración propia 
La tabla 4 muestra los resultados del indicador bajos costos, conformados por dos criterios que son: costos por producto y costos por desperdicios. En cada uno de los dos criterios del indicador el sistema ISO 9000 de las empresas tienen un impacto positivo en las empresas de estudio. Se destaca en los dos criterios que conforman el indicador la empresa CLX con los siguientes resultados: costos por producto (12\%) y PLB en costos por desperdicios $(33 \%)$.

La tabla 5, muestra los resultados del indicador racionalidad económica conformados por dos criterios que son: utilidad neta y el EVA. En cada uno de los dos criterios del indicador el sistema ISO 9000 de las empresas tienen un impacto positivo en las empresas de estudio.

Se destaca en los dos criterios que conforman el indicador las empresas CLX y BFM con los siguientes resultados: utilidad neta (36\%), CLX y el EVA (23\%) de BFM.

La tabla 6, muestra los resultados del indicador internacionalismo conformado por unos criterios que explican dicho concepto. En este indicador el sistema ISO 9000 manifiesta un impacto positivo en las empresas de estudio. Se destaca la empresa BFM con un incremento positivo del $65 \%$.

Tabla 4. Efecto de la innovación organizacional en el producto

\begin{tabular}{|c|c|c|c|c|}
\hline Indicador & \multicolumn{4}{|c|}{ BAJO COSTOS (Procesos-Producto) } \\
\hline & & IMPACTO $(\%)$ & & \\
\hline \multirow{5}{*}{$\begin{array}{l}\begin{array}{l}\text { Costos } \\
\text { producto }\end{array} \\
\end{array}$} & Empresa & Incremento & Disminución & Permanece \\
\hline & PPC & & 5 & \\
\hline & BFM & & 9 & \\
\hline & PLB & & 4 & \\
\hline & CLX & & 12 & \\
\hline \multicolumn{5}{|c|}{ IMPACTO } \\
\hline \multirow{5}{*}{$\begin{array}{c}\text { Costos por } \\
\text { desperdicios } \\
\end{array}$} & Empresa & Incremento & Disminución & Permanece \\
\hline & PPC & & 12 & \\
\hline & BFM & & 8 & \\
\hline & PLB & & 33 & \\
\hline & CLX & & 14 & \\
\hline
\end{tabular}

Fuente. Elaboración propia

Tabla 5. Efecto de la innovación organizacional en la racionalidad económica

\begin{tabular}{|l|l|l|l|l|}
\hline Indicador & \multicolumn{5}{|c|}{ RACIONALIDAD ECONÓMICA } \\
\hline & & EFECTO & & \\
\hline Utilidad neta & Empresa & Incremento & Disminución & Permanece \\
\hline & PPC & 28 & & \\
\hline & BFM & 32 & & \\
\hline & PLB & 17 & & \\
\hline & CLX & 36 & & \\
\hline & \multicolumn{5}{|c|}{ IMPACTO } & Permanece \\
\hline & Empresa & Incremento & Disminución & \\
\hline & PPC & 15 & & \\
\hline & BFM & 23 & & \\
\hline & PLB & 15 & & \\
\hline & CLX & 16 & & \\
\hline
\end{tabular}

Fuente. Elaboración propia 
Tabla 6. Efecto de la innovación organizacional en el internacionalismo

\begin{tabular}{|l|l|l|l|l|}
\hline Indicador & \multicolumn{4}{|c|}{$\begin{array}{c}\text { INTERNACIONALISMO } \\
\text { (Adecuación entorno) }\end{array}$} \\
\hline \multicolumn{5}{|c|}{ EFECTO } \\
\hline Clientes internacionales & Empresa & Incremento & Disminución & Permanece \\
\hline & PPC & 36 & & \\
\hline & BFM & 65 & & \\
\hline & PLB & 20 & & \\
\hline & CLX & 15 & & \\
\hline
\end{tabular}

Fuente. Elaboración propia

Se refleja un impacto positivo del sistema de calidad ISO 9000 en las empresas de estudio teniendo en cuenta indicadores de productividad y competitividad de las mismas.

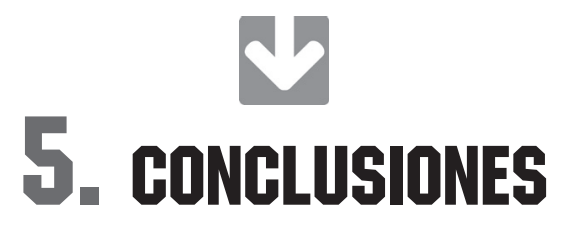

continuación, se presentan los principales hallazgos encontrados en la investigación y que aportan o validan la literatura sobre la innovación organizacional en las empresas y su relación con la productividad y la calidad. Estos aportes son los siguientes:

$\Rightarrow$ La importancia de la innovación como estrategia para el aumento de la productividad y competitividad de las empresas.

$\rightarrow$ La existencia de manera estructural en las empresas de elementos claves que potencializan la capacidad de innovación de las mismas.

$\Rightarrow$ Los sistemas de calidad y su relación con la innovación organizacional en las empresas, permitiendo mostrar un sistema de calidad como innovación organizacional que impacta positivamente en la calidad y por tanto, en la productividad de las empresas.

$\Rightarrow$ Los sistemas de dirección de las empresas son medios esenciales que permiten mejorar la capacidad de innovación; a su vez, son innovaciones organizacionales. En este estudio se puede apreciar cómo la planeación estratégica y el sistema de gestión de la calidad y su mantenimiento se convierten en un campo adecuado para implementar la cultura de la innovación.

$\Rightarrow$ La alineación de la estrategia principal de la empresa con la innovación es un paso firme que aporta a la capacidad de innovación y se enmarca dentro de las prácticas de mejoras en la estructura como innovación organizacional.

$\Rightarrow$ La orientación de la empresa a lograr una mejor atención de sus clientes con un enfoque claro hacia la calidad de todos sus productos y procesos. Esto resalta y reafirma lo que dice la literatura al respecto. 


\section{BIBLIOGRAFí́}

Abernathy, W. Y. Clark, K. (1985). Innovation: Mapping the Winds of Creative Destruction. Research Policy, 14.

Afuach, A. (1999). La dinámica de la innovación organizacional: un nuevo enfoque para lograr ventaja competitiva. OXFORD University Press. México.

Andrews, C. (1998). Enviromental Business Strategic: Corporate Leaders Perceptions. Society and Natural Resource, 11.

Ambruster, H.; Kinkel, S. y Maloca, S. (2005). Techno-Organisational Innovation in the European Manufacturing Industry. European Manufacturing Survey No. 1.

Archibugi, D. y Michie, J. (1997). Technology, globalization and Economic Perfomance. Cambridge University Press.

Argyris, C. (1985). Strategy, Change and Defense Routine. Pitman Publishing.

Avermaete, T. y otros (2003). Determinants of Inovation in Small Food Firms. European Journal of Innovation Manegement, 6, 1.

Bailey, A. y Jhonson, G. (1992). An Integrated Exploration of strategic Decision Making. Documento presentado en la Conferencia Internacional Strategic Management Society. Londres.

Barley, S. (1990). Images of Imaging: Notes on Doing Longitudinal Field Work. Organizational Science, 1.

Barnett, W. y Carroll, G. (1995). Modeling Internal Organizational Change. Annual Review of Sociology, 21.

Barragan, A Y Zubieta, J (2006). La Administración de la Calidad como generadora de innovación dentro de las organizaciones. Ponencia en: I Congreso Iberoamericano de Ciencia, Sociedad e Innovación CTS+I. 19 al 23 de junio 2006. México.

Bonache Pérez, J. (1999). El estudio de casos como estrategia de construcción teórica: características, criticas y defensas. Cuadernos de Economía y Dirección de la Empresa, 3.

Camagni, R. (1991). Innovation Networks: Spatial Perspective. Belhaven Press, London.

Camagni, R. y Capello, R. (1997). Innovation and Performance of SMEs in Italy: The Relevance of Spatial Aspects. ESRC Centre for Business Research University Cambridge, 60.

Camisón, C. y otros (2003). Marco conceptual de la relación entre la innovación y tamaño organizativo. Madrid, 19.

CIDEM (2004). Guía de Gestión de la Innovación. Barcelona.

Chaminade, C. y Hanno, R. (2003). Social Capital and Innovation in SMES: A New Model of Innovation? Evidence and Discusion. ALTEC, X Seminario Latinoamericano de Gestión Tecnológica, 2003, Méjico D.F. 
Comisión Europea (1995). Libro Verde de la Innovación. Comisión Europea. Bruselas.

Comisión Europea (2001). La innovación en una economía del conocimiento. Bruselas.

Comisión Europea (2002). Innovation Tomorrow. Comision Europea Bruselas.

Comisión Europea (2004). European Innovation Scoreboard 2004 Comparative Analysis of Innovation Performance. Commission of the European Communities.

Comision Europea (2004). Innovation Management and the Knowledge-Driving Economy. Bruselas.

Cooke, P. y Morgan, K. (1994). The Regional Innovation System in Baden Wurttemberg. International Journal of Technology, 9 .

Chalmers, A. (1992). La ciencia y cómo se elabora. Siglo XXI, Madrid.

Da Cuhna, C. (2004). Diseño de un modelo de evaluación competitiva. Una aplicación al sector plástico de Barranquilla (Colombia). Tesis Doctoral Universidad de Valencia.

Daft, R. (1992). Organizational Theory and Design. St. Paul, MN, West.

Damanpour, F. (1991). Organizational innovation: A Meta-Analysis of Effect of Determinants and Moderators. Academy of Management Journal, 34.

Damanpour, F.; Kathryn, A. y Evan, W. (1989). The Relationship between Types of Innovation of Management Studies 26.

De Toni, A.; Filippini, R. y Forza, C. (1992). Manufacturing Strategy in Global Markets: an Operations Management Model. International Journal of Operations \& Production Management. Vol. 12 No. 4.

Dogson, M. y Rothwell, R. (1994). The Handbook of Industrial Innovation. Editado por Edawrd Elgar.

Dogson, M. (1995). Organizational Learning: A Review of Some Literatures. Organization Studies, 14,3.

Dogson, M. (2000). The Management of Technological Innovation: an international and strategic approach. Oxford University Press. New York.

Drucker, P. (1986). La innovación y el empresario innovador. La práctica y los principios. Ediciones Apóstrofe. Barcelona (España).

Drucker, P. (1998). The Discipline of Innovation. Harvard Business Review.

Feeny, S. y Rogers, M. (2001). Innovation and Performance: Benchmarking Australian Firms. Melbourne Institute Working Paper No. 7/01.

Fernández, E. (1996). Innovación, tecnología y alianzas estratégicas: factores clave de la competencia. Editorial Civitas. Madrid. 
Fernández, E.; Montes, J. Y Vásquez, C. (1996). Factores de competitividad en la pequeña y mediana empresa. Inversión en activos intangibles, tamaño y límites de crecimiento. Economía Industrial, 310 IV.

Frías, J. (2006). La Tercera Edición del Manual de Oslo amplía el concepto de innovación a la de carácter no tecnológico. Revista Economía Industrial, 360.

Gibson, J. ; Ivancevich, J. Y Donnelly, J. (1996). Las organizaciones: comportamiento, estructura y proceso. Irwin, Octava edición. Madrid.

Gobierno de Australia (2003). Main Report-Mapping Australian Science and Innovation.

Grant, R. (1996). Prospering in Dynamically-Competitive Environments: Organizational Capability as Knowledge Integration. En: Organization Science, 7,4.

GranT R. (1997). The Knowledge Based View of the Firm: Implications for Managements Practice. Long Range Planning, 30, 3 .

Hamel, G. (2000). Liderando la revolución. Ediciones Gestión 2000. Barcelona.

Hagedoorn, J. y Duyster P. (2002). External Sources of Innovative Capabilities: The Preference for Strategic Allienaces or Merges and Acquisitions. Journals of Management Studies, 39.

Hair, J. y otros (2001). Análisis multivariante. Pearson.

Harenstam, A. y Bejerot, E. (2004). Multilevel analyses of organizational change and working conditions in public and private sector. European Journal of Work and Organizational Psychology , 13, 3.

Hayes, R; Wheelwright, S. y Clark, K. (1988). Dynamic Manufacturing: Creating the Learning Organization. The Free Press New York, NY.

Henderson, H. y Clark, K. (1990). Architectural Innovation: The Reconfiguration of Existing Products Technology and The Failure of Established Firms. Administrative Science Quartely, 35.

Hodgson, G. (1998). What is the Essence of Institucional Economics. Journal of Economic Literature, 36.

Holzl W.; Reintaller, A. y Windrum, P. (2005). Organizational Innovation, Information Technology and Outsourcing to Business Services. MERIT-Infonomics Memorandum Series.

Huber, G. y Glick, W. (1995). Organizational Change and Redesign, Ideas and Insights for Improving Performance. Oxford University Press.

Huergo, E. y Moreno, L. (2004). Innovación y el crecimiento de la productividad en España. Ekonomiaz No. 56, 2 Cuatrimestre.

Jacob, M.; Tintore, J. y Torres, X. (2001). Innovación en servicios. Informe del proyecto: Innovación en el sector turístico balear. Análisis prospectivo de tecnologías. Proyecto financiado por la Fundación COTEC para la Innovación Tecnológica. Madrid. 
Jacob, M.; et al (2004). Pautas de innovación en el sector turístico balear. Proyecto Financiado por la Fundación COTEC para la Innovación Tecnológica. Madrid.

Kline, S. y Rosenberg, N. (1986). An Overview of Innovation, in R. Landau and N. Rosemberg. The Positive Sun Strategic. National Academy Press, Washington.

Lam, A. (2004). Organizational Innovation. BSBM Working Papers Vol. 1 no. 2. Brunel Business School, Brunel University, Uxbridge.

Lam, A. y otros (2004). Organizational Innovations. The Oxford Handbook of Innovation. Oxford University Press.

Langlois, R. y Robertson, P. (1995). Firms, Markets and Economic Change: A Dynamic Theory of Business Institutions. Routledge (UK).

Larios, F (1999). Innovación ¿Factor de competitividad? Madrimasd, 2.

Lawrence, P. Y Lorsch, J. (1967). Organization and Environment, Cambridge, Ma., División of Research, Graduate School of Business Administration, Harvard University.

Lengrand, L. (2002). Innovation Tomorrow: Innovation Policy and the Regulatory Framework. Making Innovation an Integral Part of Broader Structural Agenda. European Communities.

Lynch, L. (2007). The Adoption and Diffusion of Organizational Innovation: Evidence for the U.S. Economy. Institute for the Study of Labor (IZA). Paper No. 2819. Bonn, Alemania.

Lopez, S. (2003). Innovación tecnológica en PYMES. Un estudio comparativo entre Barranquilla y el Valle de Mondragón. Tesis Doctoral Universidad de Mondragón ETEO.

Lorsh, J. (1986). Managing Culture: The Invisible Barrier to Strategic Change. California Management Review, 28.

Lundvall, B. (1992). National Systems of Innovation Towards: A Theory of Innovation and Interactive Learning. Frances Printer. London.

Manual de Bogotá (2001). Normalización de Indicadores de Innovación Tecnológica en América Latina y el Caribe. Red Iberoamericana de Ciencia y Tecnología (RICYT), Organización de Estados Americanos (OEA), Programa CYTED.

Manual de Bogotá (2004). Normalización de Indicadores de Innovación Tecnológica en América Latina y el Caribe (Revisión). Red Iberoamericana de Ciencia y Tecnología (RICYT), Organización de Estados Americanos (OEA), Programa CYTED.

Marklund, G. (2000). Indicators of Innovation Activities in Services. En Services and the Knowledge-Based Economy, M. Boden e I. Miles (editores). Science, Technology and the International Political Economy Series, Series editor: J. de la Mothe, pp. 86-108. Continuum,Londres, Gran Bretaña.

Maxwell, J. (1996). Qualitative Research Design: An Interactive Approach. Sage Publications.

Mertens, L. (1997). Competencia laboral, sistemas, surgimiento y modelos. Cinterfor - OIT. Montevideo. 
Medina, C. y Espinosa, M. (2000). La innovación en las organizaciones modernas. Revista Gestión y Estrategia, Universidad Autónoma de México, 5.

Miles, I. (1995). Services Innovation, Statistical and Conceptual Issues. Working Group on Innovation and Technology Policy, OCDE (Doc. DSTI/EAS/STP/NESTI (95)12. Paris, Francia.

Miles, I. (2003). Innovation in Services. Policy Research in Engineering Science and Technology. University of Manchester. TEARI, 16.

Miller, D. (1987). The Structural an Enviromental Correlates of Business Strategy. Strategic Management Journal, 8.

Morcillo, P. (1997). Dirección estratégica de la tecnología e ennovación. Un enfoque de competencias. Editorial Civitas, Madrid.

Nadler, D. y Tushman, M. (1999). El diseño de la organización como arma competitiva. El poder de la arquitectura organizacional. OXFORD University Press.

Neely, A. y Hii, J. (1998). Innovation and Business Perfomance. A Literature Review. The Judge Institute of Management Studies, University of Cambridge.

Nelson, R. y Winter, S. (1982). An Evolutionary Theoric Economic Change. Harvard University press, Cambridge, Massachusetts.

Nelson, R. (1993). National Innovation Systems. A Comparative Analysis. Oxford Press University.

Nelson, R. y Sampat, B. (2001). Making Sense of Institutions as a Factor in Economic Perfomance. Journal of Economic Behavior and Organization, 44.

Neely, A.; et al.,. (2001). A Framework for Analysinng Business Perfomance, Firm Innovation and Related Contextual Factors: Perceptions of Managers and Policy Makers in Two Europeans Regions. Integrated Manufacturing Systems 12/2. MCB University Press.

Organisation for Economic Co-Operation And Development-Ocde (1993). Frascati Manual. OCDE.

OCDE (1997). Oslo Manual: Proposed Guidelines for Collecting and Interpreting Technological Innovation Data, 2nd Edition. OCDE Publications, Paris.

OCDE (2005). Oslo Manual: Guidelines for Collecting and Interpreting Innovation, 3rd Edition. OECD Publications, Paris.

Palacios, B. y Galvan, S. (2003). Gestión del conocimiento y la estrategia. Madrimasd, 19.

Panda, H. y Ramanathan, K. (1996). Technological Capability Assessment of a Firms in the Electricity Sector. Technovation, 16, 10.

Pavón, J. e Hidalgo, A. (1999). Gestión de la innovación: un enfoque estratégico. Ediciones Pirámide. Madrid. 
Perdomo, J. y otros (2004). La gestión de la calidad total como un antecedente de la capacidad de innovación empresarial. Documento de trabajo 09/04. Departamento de Administración y Economía de la Empresa. Universidad de Salamanca.

Porter, M. (2003). Ser competitivos. Nuevas aportaciones y conclusiones. Deusto. España.

Porter, M. (2006). Estrategia y ventaja competitiva. Ediciones Deusto.

Roberts, E. (1996). Gestión de la innovación tecnológica. Editado COTEC. Madrid.

Robinson, W. (1951). The Logical structure of Analitic Induction. American Sociological Review, 16.

Roger, E. (2003). Diffusion of innovations. Quinta edicion. Simon y Schuster. New York.

Rosenbloom, R. Y Christensen, C. (1994). Technological Discontinuities, Organizational Capabilities, and Strategic Commitments. Industrial and Corporate Change, 3,3.

Rotember, J. y Salomer, G. (2000). Visionaries, Managers and Strategic Direction. Journal of Economics, 31, 4.

Salavou, H.; et al. (2004). Organizational Innovation in SMEs. The Importance of strategic and Competitive structure. European Journal of Marketing, 9/10.

SAnchez, M. y Castrillo, R. (2006). La tercera edición del Manual de Oslo: cambios e implicaciones. una perspectiva de capital intelectual. Madrimasd, 35.

Schrage, M. (2001). Juego serio. Cómo las mejores compañías usan la simulación para innovar. Oxford University Press.

Schmidt, T. y Rammer, C. (2007). Non-technological and Technological Innovation: Strange Bedfellows. Discussion Paper No. 07-052. Centre European Economic Research (ZEW). Mannheim (Alemania).

Solleiro, J. y Castañon, R. (2004). Competitividad y sistemas de innovación: los retos para la inserción de México en el contexto global. Sala de Lectura CTS+I de la OEI.

Sosa, S. (2003). La génesis y el desarrollo del cambio estratégico: un enfoque dinámico basado en el momentum organoizativo. Tesis Doctoral Departamento de Economía y Dirección de Empresas Universidad de Gran Canaria.

Sumanth, D. (1990). Ingeniería y Administración de la Productividad: medición, evaluación, planeación y mejoramiento de la productividad en las organizaciones de manufactura y servicio. McGraw Hill/Interamericana, México.

Subramanian, A. y Nilakanta, S. (1996). Organisational Innovativeness: Exploring the Relationship Between Organisational Determinanst of Innovation, Types of Innovations, and Measures of Organisational Perfomance. Omega International Journal of Management Science 6.

Tornatzky, L. y Fleischer, M. (1990). The Process of Technological Innovation. Lexington, MA: Lexington Books.

Tsan E.W. K. (1997). Organizacional learning and the learning organization: A dichotomy between descriptive and prescriptive research. Human Relations, 50, 1. 
Van de Ven, A. (1992). Suggestions for Studying Strategic Process: A Researc Note. Strategic Management Journal, 13.

Van de Ven, A., (2004). Organizational Change. Blackwell Publishers, forthcoming. London.

Van de Ven, A. y Huber, G. (1990). Longitudinal Field Research Methods for Studying Processes of Organizacional Change. Organizational Science, 1.

Van de Ven, A. y Poole, M. (2004). Handbook of Organizacional Change and Innovation. Oxford University Press.

Van de Ven, A. et al. (2000). Research the Management of Innovation. The Minnesota Studies. Oxford University Press.

Van de Ven, A. y otros (2001). El viaje de la innovación: el desarrollo de una cultura organizacional para innovar. OXFORD University Press.

Varela, R. (2001). Innovación empresarial: arte y ciencia en la creación de empresas. Pearson Educación de Colombia. Von Hippel, E. (1988). The Sources of Innovation. Oxford University Press.

Yin, R (1994). Case Study Research. Design and Methods. Sage Publications.

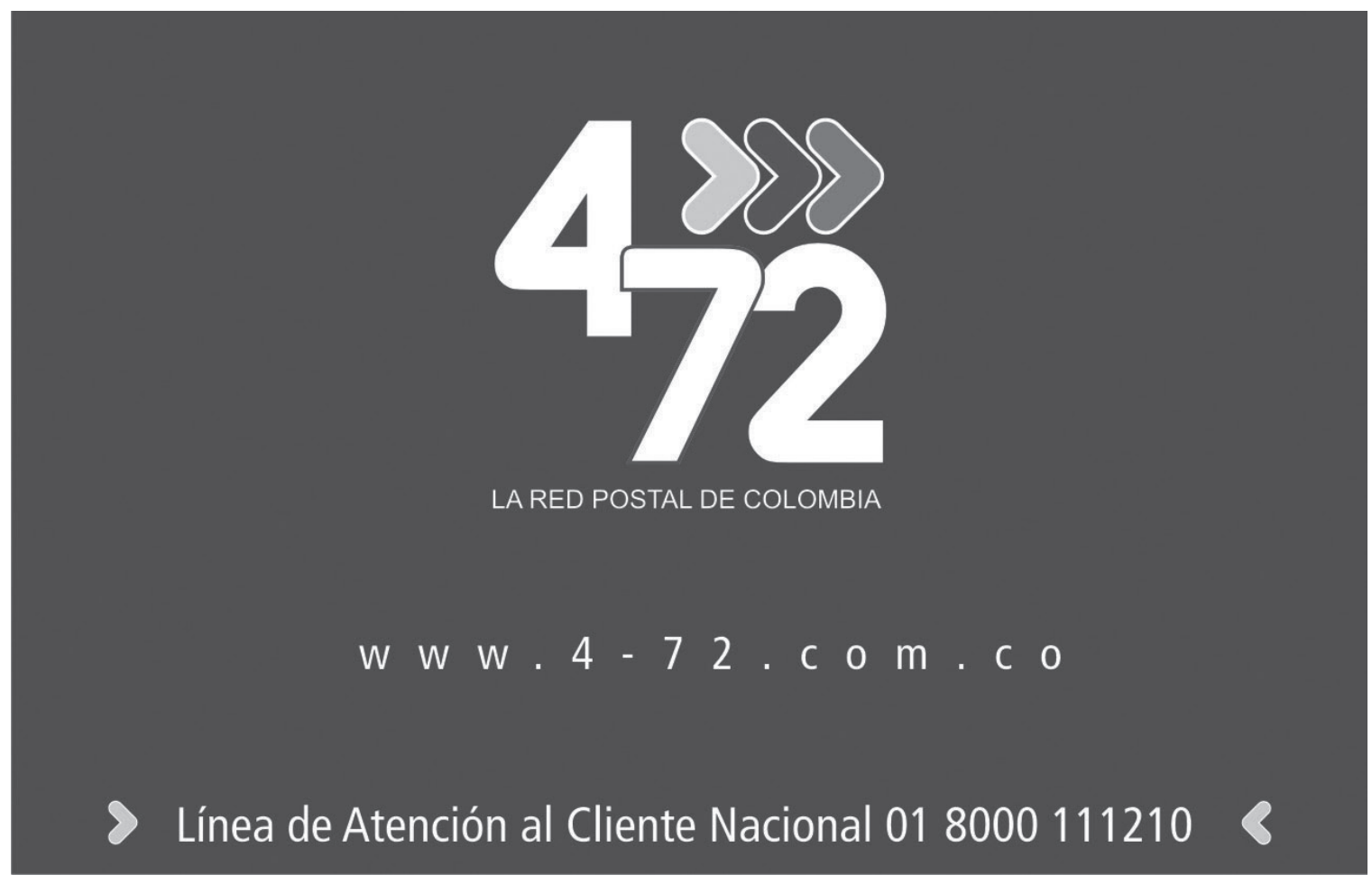

